\title{
Realizing nonadiabatic holonomic quantum computation beyond the three-level setting
}

\author{
G. F. Xu, ${ }^{1}$ P. Z. Zhao, ${ }^{1}$ Erik Sjöqvist $\odot,{ }^{2, *}$ and D. M. Tong ${ }^{1, \dagger}$ \\ ${ }^{1}$ Department of Physics, Shandong University, Jinan 250100, China \\ ${ }^{2}$ Department of Physics and Astronomy, Uppsala University, Box 516, Se-751 20 Uppsala, Sweden
}

(Received 13 May 2020; accepted 28 April 2021; published 13 May 2021)

\begin{abstract}
Nonadiabatic holonomic quantum computation (NHQC) provides a method to implement error resilient gates and that has attracted considerable attention recently. Since it was proposed, three-level $\Lambda$ systems have become the typical building block for NHQC and a number of NHQC schemes have been developed based on such systems. In this paper, we investigate the realization of NHQC beyond the standard three-level setting. The central idea of our proposal is to improve NHQC by enlarging the Hilbert space of the building block system and letting it have a bipartite graph structure in order to ensure purely holonomic evolution. Our proposal not only improves conventional qubit-based NHQC by efficiently reducing its duration, but also provides implementations of qudit-based NHQC. Therefore, our proposal provides a further development of NHQC that can contribute significantly to the physical realization of efficient quantum information processors.
\end{abstract}

DOI: 10.1103/PhysRevA.103.052605

\section{INTRODUCTION}

Quantum computation is realized by using quantum principles and therefore it processes information differently from classical computation [1]. By utilizing quantum parallelism, quantum computation enables efficient solutions of certain computational tasks, like factoring large integers [2] and searching unsorted databases [3]. However, practical quantum computation is still challenging and requires further theoretical and technological development. It is known that the main obstacle to practical quantum computation is errors caused by either inaccurate manipulation of quantum systems or interaction with their environment. To address this problem, various error-resilient models have been proposed. Among these error-resilient models, holonomic quantum computation plays an important role.

Nonadiabatic holonomic quantum computation (NHQC) $[4,5]$ is realized by using nonadiabatic non-Abelian geometric phases [6]. Since these phases depend only on the global nature of evolution paths, but not on evolution details, NHQC provides a geometry-based approach for implementing errorresilient quantum gates. The most attractive feature of NHQC is that it can be performed at high speed and simultaneously preserves the geometric robustness against errors. Moreover, its robustness can be widened by combining it with other error reducing methods [5,7-12]. Due to these features, NHQC

\footnotetext{
*erik.sjoqvist@physics.uu.se

†tdm@sdu.edu.cn
}

Published by the American Physical Society under the terms of the Creative Commons Attribution 4.0 International license. Further distribution of this work must maintain attribution to the author(s) and the published article's title, journal citation, and DOI. Funded by Bibsam. has received considerable attention and various nonadiabatic holonomic schemes have been put forward [4,5,7-34]. In particular, NHQC has been demonstrated experimentally in circuit QED [13,27,28,30,31], NMR [11,14,23], NV centers in diamond [15,16,24,25], and trapped ions [33].

Three-level $\Lambda$ systems have become the typical building block for NHQC since it was proposed. While impressive progress has been achieved by using this setting, a natural and important topic is whether one can benefit from realizing NHQC with other building block systems. The investigation of this topic not only enriches the theory of NHQC, but may also provide a different way to refine NHQC. Furthermore, with the development of quantum technologies, the ability to control multilevel quantum systems has been improved significantly. Recently, diverse experimental platforms including photons, NV centers, trapped ions, and superconducting circuits have begun to explore multilevel-based quantum information processing. In particular, the challenges of controlling multilevel systems have been shown to set no fundamental limitations for high-fidelity multilevel-based quantum gates [35-50]. This technological progress also greatly encourages us to investigate the topic of realizing NHQC with other building block systems.

In this paper, we investigate the realization of NHQC beyond the standard three-level setting. Specifically, we improve NHQC by enlarging the Hilbert space of the building block system and letting it have a bipartite graph structure. In this way, not only conventional qubit-based NHQC can be improved, but also the possibility to realize universal qudit-based NHQC is provided. We base our proposal on trapped ions, for which all relevant technologies, such as initialization, readout, and controllable interactions, are achievable for designing multilevel-based quantum computation. Our proposal indicates that enlarging the Hilbert space of the building block system and letting it have an appropriate energy-level structure can be a promising direction to develop further NHQC. In 


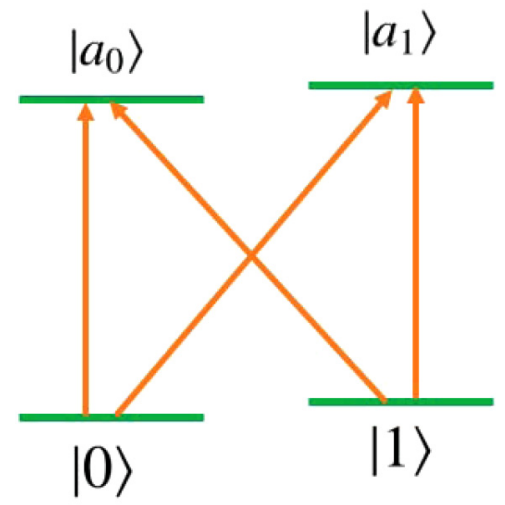

FIG. 1. States in $V_{1}=\{|0\rangle,|1\rangle\}$ span the computational subspace and those in $V_{2}=\left\{\left|a_{0}\right\rangle,\left|a_{1}\right\rangle\right\}$ are used as auxiliary states.

this way, our proposal contributes to the physical realization of efficient and robust quantum information processors.

\section{PROPOSAL}

We now demonstrate the first merit of enlarging the Hilbert space of the building block system: the duration of NHQC can be efficiently reduced. Circuit-based quantum computation, including NHQC, uses the three basic components, one-, two-, and multiqubit gates, to process information. As the number of operation steps to realize these components decreases, the duration of the computation can be reduced. A shorter duration corresponds to a reduced exposure to errors caused by decoherence and therefore an increased robustness and precision of the computation. Note that quantum information is entering noisy intermediate-scale quantum era, in which quantum computers lack the resources for full fault tolerance and therefore can only support computation of short duration. This makes the reduction of computation duration a pertinent issue.

To reduce the duration of NHQC, one may use the building block system having the structure in Fig. 1. This system exhibits a bipartite graph structure, i.e., its energy levels are partitioned into two sets $V_{1}=\{|0\rangle,|1\rangle\}$ and $V_{2}=\left\{\left|a_{0}\right\rangle,\left|a_{1}\right\rangle\right\}$, and no transitions exist within each set. The states in $V_{1}$ span the computational subspace and those in $V_{2}$ are auxiliary states. The key point with the bipartite structure is that it allows us to perform nonadiabatic gates that are purely holonomic since there are no transitions within the computational subspace $V_{1}$.

We begin by showing the realization of holonomic onequbit gates with the system having the structure in Fig. 1. One way for the realization is as follows. Consider one ion and apply resonant laser fields to drive the transitions between one of the auxiliary states, say $\left|a_{0}\right\rangle$, and the computational states $|0\rangle$ and $|1\rangle$. In this way, one designs the Hamiltonian

$$
H_{1}(t)=\Omega_{1}^{0}(t)\left|a_{0}\right\rangle\left\langle 0\left|+\Omega_{1}^{1}(t)\right| a_{0}\right\rangle\langle 1|+\text { H.c. },
$$

where $\Omega_{1}^{0}(t)$ and $\Omega_{1}^{1}(t)$ are Rabi frequencies chosen such that $\Omega_{1}^{0}(t) / \Omega_{1}^{1}(t)$ is time independent. This makes $H_{1}(t)$ commute with itself at different times, which is an important ingredient for guaranteeing the holonomic feature, as it ensures that the dynamical phases $\int_{0}^{t}\left\langle k\left|U^{\dagger}\left(t^{\prime}, 0\right) H_{1}\left(t^{\prime}\right) U\left(t^{\prime}, 0\right)\right| l\right\rangle d t^{\prime}$, with $k, l=0,1$ and $U\left(t^{\prime}, 0\right)$ the time evolution operator, all vanish (parallel transport). The Hamiltonian $H_{1}(t)$ describes an effective three-level $\Lambda$ system, which is known to be sufficient to realize arbitrary holonomic one-qubit gates [4]. Thus the system in Fig. 1 is universal on the single qubit level.

We now show the benefits of using the system to realize holonomic two-qubit gates. Consider two ions each of which has the level structure shown in Fig. 1. We couple these two ions by bichromatic lasers that drive sideband transitions between the computational and auxiliary states. Under large detuning condition, the single ion transitions are strongly suppressed, while only the double ion transitions are allowed due to exchange of vibrational energy between the ions. In this way, the coupling between the computational state $|i j\rangle$ and the auxiliary state $\left|a_{m} a_{n}\right\rangle$ can be designed, where $i, j, m, n \in$ $\{0,1\}[51,52]$. (See Appendix A for details.) Based on the above coupling mechanism, one can realize the Hamiltonian

$$
H_{2}(t)=\Omega_{2}^{0}(t)\left|a_{0} a_{0}\right\rangle\left\langle\phi_{0}\left|+\Omega_{2}^{1}(t)\right| a_{1} a_{1}\right\rangle\left\langle\phi_{1}\right|+\text { H.c. }
$$

where $\Omega_{2}^{0}(t)$ and $\Omega_{2}^{1}(t)$ are Rabi frequencies and $\left|\phi_{0}\right\rangle$ and $\left|\phi_{1}\right\rangle$ are states residing in the computational subspace spanned by $|10\rangle$ and $|11\rangle$. Note that, contrary to the realization of the holonomic one-qubit gate by means of $H_{1}(t)$ above, the ratio of the Rabi frequencies is allowed to be time dependent, since $\left|\phi_{0}\right\rangle$ and $\left|\phi_{1}\right\rangle$ couple to orthogonal auxiliary states $\left|a_{0} a_{0}\right\rangle$ and $\left|a_{1} a_{1}\right\rangle$. We choose the coupling parameters so that $\left|\phi_{0}\right\rangle$ and $\left|\phi_{1}\right\rangle$ become mutually orthogonal and divide the evolution into two intervals $0 \leqslant t<\tau$ and $\tau \leqslant t \leqslant T$. We divide the run time $T$ so as to satisfy $\int_{t_{0}}^{t_{1}}\left|\Omega_{2}^{0}(t)\right| d t=\int_{t_{0}}^{t_{1}}\left|\Omega_{2}^{1}(t)\right| d t=\pi / 2$ with $t_{0}=0, t_{1}=\tau$ or $t_{0}=\tau, t_{1}=T$, and set the phase of $\Omega_{2}^{0}(t)$ [and $\Omega_{2}^{1}(t)$ ] to be different constants for $0 \leqslant t<\tau$ and $\tau \leqslant t \leqslant T$. One thereby obtains the gate

$$
U_{2}=|00\rangle\langle 00|+| 01\rangle\left\langle 01\left|+e^{i \gamma_{0}}\right| \phi_{0}\right\rangle\left\langle\phi_{0}\left|+e^{i \gamma_{1}}\right| \phi_{1}\right\rangle\left\langle\phi_{1}\right|,
$$

where $\gamma_{0}$ and $\gamma_{1}$ are relative phases that can be controlled by varying the stepwise phase changes of $\Omega_{2}^{0}(t)$ and $\Omega_{2}^{1}(t)$ during the evolution. The condition the evolution time satisfies is important and if it does not hold there will be a leakage to the subspace spanned by the auxiliary states. We also note that, while this condition is important, previous experimental realizations of NHQC have shown that it can be met with high precision. The above realization can be further generalized with the bichromatic coupling mechanism. Specifically, one can design the Hamiltonian

$$
\begin{aligned}
H_{3}(t)= & \Omega_{3}^{a}(t)\left|a_{0} a_{0}\right\rangle\left\langle\phi_{a}\left|+\Omega_{3}^{b}(t)\right| a_{1} a_{1}\right\rangle\left\langle\phi_{b}\right| \\
& +\Omega_{3}^{c}(t)\left|a_{0} a_{1}\right\rangle\left\langle\phi_{c}\right|+\text { H.c. },
\end{aligned}
$$

where $\Omega_{3}^{a}(t), \Omega_{3}^{b}(t)$, and $\Omega_{3}^{c}(t)$ are Rabi frequencies, and $\left|\phi_{a}\right\rangle$, $\left|\phi_{b}\right\rangle$, and $\left|\phi_{c}\right\rangle$ are states residing in the space spanned by $|00\rangle$, $|01\rangle,|10\rangle$, and $|11\rangle$. Similar to realizing $U_{2}$, we choose coupling parameters so that $\left|\phi_{a}\right\rangle,\left|\phi_{b}\right\rangle$, and $\left|\phi_{c}\right\rangle$ become mutually orthogonal and divide the evolution into two time intervals. Moreover, the phases of $\Omega_{3}^{a}(t), \Omega_{3}^{b}(t)$, and $\Omega_{3}^{c}(t)$ are different constants for different intervals. In this way, one realizes the gate

$$
U_{3}=\left|\phi_{\perp}\right\rangle\left\langle\phi_{\perp}\left|+e^{i \gamma_{a}}\right| \phi_{a}\right\rangle\left\langle\phi_{a}\left|+e^{i \gamma_{b}}\right| \phi_{b}\right\rangle\left\langle\phi_{b}\left|+e^{i \gamma_{c}}\right| \phi_{c}\right\rangle\left\langle\phi_{c}\right|,
$$


where $\gamma_{a}, \gamma_{b}$, and $\gamma_{c}$ are variable relative phases and $\left|\phi_{\perp}\right\rangle$ is orthogonal to $\left|\phi_{a}\right\rangle,\left|\phi_{b}\right\rangle$, and $\left|\phi_{c}\right\rangle$. The gate $U_{2}$ represents arbitrary two-qubit controlled gates, while $U_{3}$ represents arbitrary two-qubit gates. The holonomic feature of $U_{2}$ and $U_{3}$ follows from the bipartite structure of the underlying fourlevel systems, but can also be verified explicitly by checking the parallel transport and cyclic conditions. In contrast, if one uses three-level ions to realize $U_{2}$ or $U_{3}$, two or more sequentially implemented gates are needed, which increases the number of operation steps of the realization and therefore the duration of the whole computation. Thus the system in Fig. 1 brings enhanced flexibility to realizing two-qubit nonadiabatic holonomic gates and therefore offers benefits to the reduction of computation duration.

Multiqubit gates can be built with gates from the universal set of one- and two-qubit gates. However, this procedure typically becomes very demanding as the number of such gates rapidly grows with the size of the computational problem. Thus finding a way to realize multiqubit gates with fewer steps is important and a key factor in reducing computation duration. Among multiqubit gates, controlled gates play a particularly prominent role. They are frequently used in various quantum algorithms and quantum error correction schemes [1-3]. We next demonstrate the benefits of using the level structure in Fig. 1 to realize holonomic multiqubit controlled gates.

The essence of our method is to separate the ions into blocks and use the freedom of the four-level system to permit operations acting on different blocks to be implemented in parallel, so that the number of operation steps can be reduced. Consider $N$ ions confined in a linear trap, each of which has the bipartite graph structure shown in Fig. 1. We divide the $N$ ions into $m \geqslant 1$ blocks and let each block $k \in\{1, \ldots, m\}$ contain $n_{k}$ ions. We consider for clarity a specific realization procedure to demonstrate our method.

Suppose there are two blocks $k=1,2$, each having six ions $\left(n_{1}=n_{2}=6\right)$ and each ion encoding a qubit. We consider the realization of a nonadiabatic holonomic controlled phase flip gate that acts on the ions of these two blocks so that $|1 \ldots 1\rangle$ is taken into $-|1 \ldots 1\rangle$, while the remaining computational states are kept unchanged. We denote the ions in each block as $1, \ldots, 6$. For block $k=1$, we first implement the transitions $|11\rangle_{1,2} \rightarrow\left|a_{0} a_{0}\right\rangle_{1,2}$ and $|01\rangle_{3,4} \rightarrow\left|a_{1} a_{1}\right\rangle_{3,4}$; secondly, we implement the transitions $\left|a_{0} 1\right\rangle_{2,4} \rightarrow\left|1 a_{0}\right\rangle_{2,4}$ and $|01\rangle_{5,6} \rightarrow\left|a_{1} a_{1}\right\rangle_{5,6}$; thirdly, we implement the transition $\left|a_{0} 1\right\rangle_{4,6} \rightarrow\left|1 a_{0}\right\rangle_{4,6}$. For block $k=2$, we first implement the transitions $|11\rangle_{1,2} \rightarrow\left|a_{0} a_{0}\right\rangle_{1,2},|01\rangle_{3,4} \rightarrow\left|a_{1} a_{1}\right\rangle_{3,4}$, and $|0\rangle_{6} \rightarrow\left|a_{1}\right\rangle_{6}$; the second and third steps are respectively the same as the second and third steps of block $k=1$. It is noteworthy that the operations acting on different blocks can be implemented in parallel. Thus only three steps are needed for the above operations. The fourth step is to couple the two blocks by implementing $\left|a_{0} a_{0}\right\rangle_{6_{1}, 6_{2}} \rightarrow-\left|a_{0} a_{0}\right\rangle_{6_{1}, 6_{2}}$ with the help of the intermediate state $|00\rangle_{6_{1}, 6_{2}}$; here, we have used the notation $\mu_{k}$ to denote ion $\mu$ of block $k$ for clarity. Finally, we implement the first three steps again but with both the implementation order and the implemented transitions reversed. Thus the realization costs 7 steps and the realized gate can be verified to yield a holonomic controlled phase gate. (See Appendix B for further details.)
The above procedure can be generalized to the case of more than two blocks. For illustrative purpose, consider three blocks $k=1,2,3$, each block containing six ions. The first three steps of blocks $k=1,2$ are the same as before, while the first three steps of block $k=3$ are the same as those of block $k=2$. Thus, because of parallelism, it costs three steps in total. The fourth step is to couple the first two blocks by implementing $\left|a_{0} a_{0}\right\rangle_{6_{1}, 6_{2}} \rightarrow|00\rangle_{6_{1}, 6_{2}}$. Next, we couple the second and third blocks by implementing $\left|0 a_{0}\right\rangle_{6_{2}, 6_{3}} \rightarrow-\left|0 a_{0}\right\rangle_{6_{2}, 6_{3}}$ with the help of state $\left|a_{0} 0\right\rangle_{6_{2}, 6_{3}}$. Finally, we repeat the first four steps but with the implementation order and the implemented transitions reversed. The above realization costs 9 steps and the realized controlled phase gate is purely holonomic. Generalizing to four such blocks requires 11 steps. In fact, the parallelism offered by four-level systems permits the blocks to contain more ions: in the three-block case, the third block can contain eight ions; in the four-block case, the third and fourth blocks can respectively contain eight and ten ions. Although the number of ions increases, the number of operation steps remain 9 and 11 , respectively.

The four-level system allows us to use 7, 9, and 11 steps to realize holonomic controlled phase gates acting on various numbers of qubits. In contrast, to achieve the same task by using three-level $\Lambda$ systems, the best known scheme needs 21, 37, and 57 steps, respectively [29]. Thus the number of steps can be reduced significantly by replacing the standard $\Lambda$ setting by the four-level building block. From the above comparison, one can also see that the reduction of the number of steps becomes more obvious with the increase of the number of blocks, i.e., the number of qubits the controlled phase gate acts on. Note that, for a practical quantum computer, the number of qubits is usually very large. In this case, the number of steps may also be saved by orders of magnitude. It is also noteworthy that, although we use a specific realization procedure, our method can be generalized to realize holonomic controlled gates acting on different numbers of qubits.

We have shown how NHQC can be improved by using a building block system with a four-level bipartite graph structure. We next take this idea one step further and show that, by enlarging the Hilbert space of the building block system and letting it have a bipartite graph structure, one can have a feasible platform to develop qudit-based NHQC, in which the holonomic gates act on $d$-dimensional quantum objects. We explicitly demonstrate the realization of qutrit-based $(d=3)$ NHQC to show this.

Before proceeding further, we briefly explain the necessity of developing qudit-based NHQC. Compared to qubit settings, qudit-based processors can store exponentially larger amounts of information and thereby provide reduction of the circuit complexity and simplification of the experimental setup [53-55]. Qudit-based processors can also enable enhancement of the algorithm efficiency, favorable error thresholds, and high-fidelity magic-state distillation [56-61]. This benefits quantum error corrections and is essential for scalable quantum computation. Particularly, qudit-based processors have recently begun to be explored on various experimental platforms [44-50]. Because of these features, developing qudit-based NHQC is of significance.

We now explicitly develop qutrit-based $(d=3)$ NHQC with ions comprising the five-level bipartite graph structure 


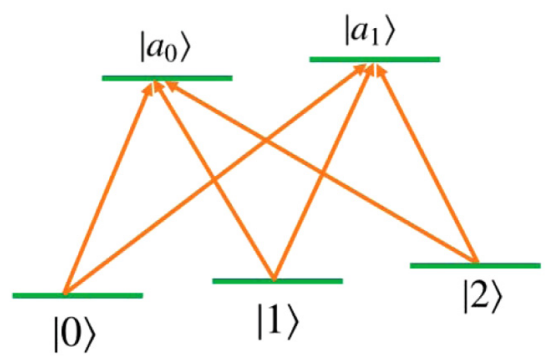

FIG. 2. States in $V_{1}=\{|0\rangle,|1\rangle,|2\rangle\}$ span the computational subspace and those in $V_{1}=\left\{\left|a_{0}\right\rangle,\left|a_{1}\right\rangle\right\}$ are used as auxiliary states.

shown in Fig. 2. The states $|0\rangle,|1\rangle,|2\rangle$ span the qutrit computational subspace and $\left|a_{0}\right\rangle,\left|a_{1}\right\rangle$ are auxiliary states. We show that the system in Fig. 2 permits us to conveniently realize not only holonomic one-qutrit and two-qutrit gates, but also holonomic multiqutrit controlled gates.

To realize holonomic one-qutrit gates, we apply resonant laser fields to drive transitions between the computational and auxiliary states, as described by the Hamiltonian $H(t)=$ $\sum_{i=0}^{2} \Omega_{0 i}(t)\left|a_{0}\right\rangle\left\langle i\left|+\sum_{j=0}^{2} \Omega_{1 j}(t)\right| a_{1}\right\rangle\langle j|+$ H.c., where $\Omega_{0 i}(t)$ and $\Omega_{1 j}(t)$ are Rabi frequencies. By letting $\Omega_{0 i}(t) / \Omega_{0 j}(t)$ and $\Omega_{1 i}(t) / \Omega_{1 j}(t)$ be constant for all pairs $i, j=0,1,2$, the Hamiltonian turns into

$$
H_{4}(t)=\Omega_{4}^{0}(t)\left|a_{0}\right\rangle\left\langle\psi_{0}\left|+\Omega_{4}^{1}(t)\right| a_{1}\right\rangle\left\langle\psi_{1}\right|+\text { H.c. },
$$

where $\Omega_{4}^{0}(t)$ and $\Omega_{4}^{1}(t)$ are common envelopes and $\left|\psi_{0}\right\rangle$ and $\left|\psi_{1}\right\rangle$ are states residing in $L=\operatorname{Span}\{|0\rangle,|1\rangle,|2\rangle\}$. By choosing the pulse areas appropriately, one can use $H_{4}(t)$ to generate

$$
U_{4}=\left|\psi_{\perp}\right\rangle\left\langle\psi_{\perp}\left|+e^{i \xi_{0}}\right| \psi_{0}\right\rangle\left\langle\psi_{0}\left|+e^{i \xi_{1}}\right| \psi_{1}\right\rangle\left\langle\psi_{1}\right|,
$$

where $\left|\psi_{0}\right\rangle,\left|\psi_{1}\right\rangle$, and $\left|\psi_{\perp}\right\rangle$ can be chosen mutually orthogonal and $\xi_{0}$ and $\xi_{1}$ are relative phases. The gate $U_{4}$ represents an arbitrary one-qutrit gate acting on $L$ and can be verified to be holonomic. Since $U_{4}$ is a gate acting on a three-dimensional Hilbert space, one needs to use two one-qubit gates and an entangling two-qubit gate to simulate it with qubit-based NHQC.

To realize holonomic two-qutrit gates, we consider two ions, each of which has the structure in Fig. 2. We use the bichromatic laser pulse mechanism to couple the two ions, just as in the realization of $H_{2}(t)$ and $H_{3}(t)$ above, and consider

$$
\begin{aligned}
H_{5}(t)= & \Omega_{5}^{a}(t)\left|a_{0} a_{0}\right\rangle\left\langle\psi_{a}\left|+\Omega_{5}^{b}(t)\right| a_{1} a_{1}\right\rangle\left\langle\psi_{b}\right| \\
& +\Omega_{5}^{c}(t)\left|a_{0} a_{1}\right\rangle\left\langle\psi_{c}\right|+\text { H.c. },
\end{aligned}
$$

where $\Omega_{5}^{a}(t), \Omega_{5}^{b}(t)$, and $\Omega_{5}^{c}(t)$ are Rabi frequencies and $\left|\psi_{a}\right\rangle$, $\left|\psi_{b}\right\rangle$, and $\left|\psi_{c}\right\rangle$ are mutually orthogonal states residing in the computational subspace $\operatorname{Span}\{|20\rangle,|21\rangle,|22\rangle\}$. By following the approach that results in $U_{2}$ and $U_{3}$ above, $H_{5}(t)$ can be used to realize the two-qutrit gate

$$
U_{5}=I_{\perp}+e^{i \xi_{a}}\left|\psi_{a}\right\rangle\left\langle\psi_{a}\left|+e^{i \xi_{b}}\right| \psi_{b}\right\rangle\left\langle\psi_{b}\left|+e^{i \xi_{c}}\right| \psi_{c}\right\rangle\left\langle\psi_{c}\right|,
$$

where $I_{\perp}$ is the identity operator acting on the space orthogonal to $\operatorname{Span}\{|20\rangle,|21\rangle,|22\rangle\}$ and $\xi_{a}, \xi_{b}$, and $\xi_{c}$ are variable relative phases. $U_{5}$ is a two-qutrit controlled gate and its holonomic feature can be verified. It acts on a nine-dimensional space so that one needs to use four qubits to simulate it with qubit-based NHQC.
We now demonstrate the realization of holonomic multiqutrit controlled gates with systems in Fig. 2. In fact, the method we used to realize holonomic multiqubit controlled gates as described above can be translated to the multiqutrit case. We use a specific realization procedure to demonstrate the basic principle. We consider realizing a controlled phase gate acting on qutrits. Here, the controlled phase gate flips the sign of $|2 \ldots 2\rangle$, while all other computational states remain unchanged. To realize this gate, we need to substitute the state $|1\rangle$ in the previous procedure with the state $|2\rangle$. For example, the transitions $|11\rangle_{1,2} \rightarrow\left|a_{0} a_{0}\right\rangle_{1,2}$ and $\left|a_{0} 1\right\rangle_{2,4} \rightarrow\left|1 a_{0}\right\rangle_{2,4}$ should be replaced by the transitions $|22\rangle_{1,2} \rightarrow\left|a_{0} a_{0}\right\rangle_{1,2}$ and $\left|a_{0} 2\right\rangle_{2,4} \rightarrow\left|2 a_{0}\right\rangle_{2,4}$, respectively. Furthermore, we need to replace the transition $|01\rangle_{\alpha, \beta} \rightarrow\left|a_{1} a_{1}\right\rangle_{\alpha, \beta}$ in the previous procedure with the two transitions $|02\rangle_{\alpha, \beta} \rightarrow\left|a_{1} a_{1}\right\rangle_{\alpha, \beta}$ and $|12\rangle_{\alpha, \beta} \rightarrow\left|a_{0} a_{1}\right\rangle_{\alpha, \beta}$. With these changes, one can realize the desired holonomic controlled phase gates acting on qutrits. The above procedure can also be generalized to realize arbitrary holonomic multiqutrit controlled gates. Thus the graph structure shown in Fig. 2 permits an efficient way to realize holonomic multiqutrit controlled gates.

We have shown that various holonomic qutrit-based gates can be conveniently realized with the system in Fig. 2, which benefits the reduction of the duration of NHQC. Moreover, since qutrits store exponentially larger amounts of information than qubits, qutrit-based NHQC has the advantages mentioned above, such as reduction of the circuit complexity, simplification of the experimental setup, etc.

The idea of our proposal is to improve NHQC by enlarging the Hilbert space of the building block system and letting it have a bipartite graph structure, and we have demonstrated the cases of four-level and five-level bipartite graph systems described in Figs. 1 and 2, respectively. We can take the idea one more step further to consider a bipartite graph system with more energy levels as the building block system for NHQC. In this case, qudit-based NHQC with larger computational space can be developed and the specific realization procedure is similar to that for realizing holonomic qutrit-based gates demonstrated above.

\section{CONCLUSION}

In this work, we propose settings for NHQC beyond the typical three-level $\Lambda$ configuration. The proposed settings have a larger Hilbert space and meanwhile a bipartite graph structure. Our results show that using the proposed settings not only improves conventional qubit-based NHQC by efficiently reducing its duration, but also provides a platform for efficiently realizing universal qudit-based NHQC. Our proposal opens up for several extensions. First, while our proposal uses bipartite graph systems, other kinds of multilevel systems may also be useful for refining NHQC. The investigation of such schemes may provide a new framework to develop NHQC. Secondly, while our proposal provides one way of realizing holonomic gates with bipartite graph multilevel systems, investigating other coupling sequences for realizing holonomic gates with such systems is worth paying attention to. Such an investigation may result in improved schemes to use bipartite graph systems for NHQC. Finally, qudit-based NHQC can be combined with other error-resilient 

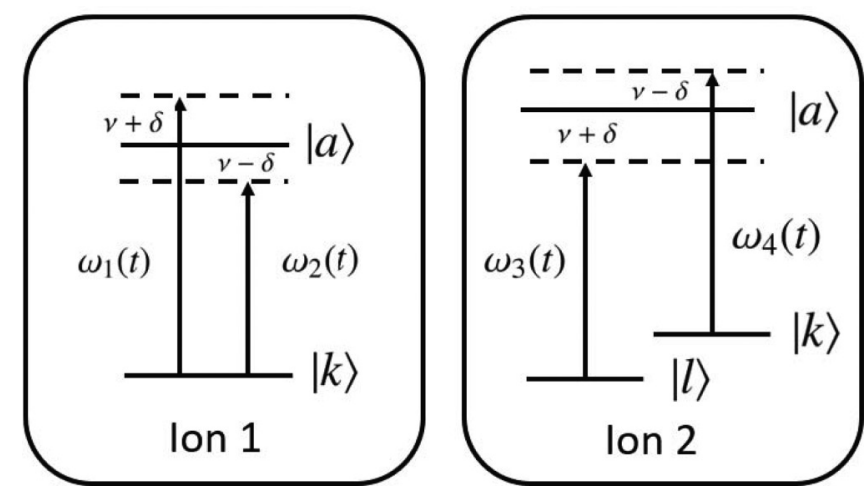

FIG. 3. Schematic for the ion-laser interaction. For simplicity, we only depict the levels that are addressed by the lasers.

methods, such as decoherence-free subspaces and noiseless subsystems, to realize quantum information processing with improved robustness features.

\section{ACKNOWLEDGMENTS}

This work was supported by the National Natural Science Foundation of China through Grant No. 11775129. P.Z.Z. acknowledges support from the National Natural Science Foundation of China through Grant No. 11947221. E.S. acknowledges support from the Swedish Research Council (VR) through Grant No. 2017-03832.

\section{APPENDIX A: COUPLING MECHANISMS}

We demonstrate two mechanisms to couple the internal states of two ions, labeled as 1 and 2, for which all the needed Hamiltonians of our proposal can be realized. Our first coupling mechanism makes use of the transition $|k\rangle \leftrightarrow|a\rangle$ of ion 1 , driven by a blue detuned laser with detuning $-(v+\delta)$ and Rabi frequency $\omega_{1}(t)$ and by a red detuned laser with detuning $(v-\delta)$ and Rabi frequency $\omega_{2}(t)$, where $|k\rangle$ is one of the computational states and $|a\rangle$ is one of the auxiliary states. Meanwhile, the transition $|l\rangle \leftrightarrow|a\rangle$ of ion 2 is driven by a red detuned laser with detuning $v+\delta$ and Rabi frequency $\omega_{3}(t)$ and $|k\rangle \leftrightarrow|a\rangle$ of ion 2 by a blue detuned laser with detuning $-(v-\delta)$ and Rabi frequency $\omega_{4}(t)$, where $|l\rangle$ is another computational state. In the above, $v$ is the phonon frequency and $\delta$ is an additional detuning. One can see the ion-laser interaction from Fig. 3. In this way, one realizes the Hamiltonian

$$
\begin{aligned}
H(t)= & i \eta \omega_{1}(t) e^{-i \delta t} b^{\dagger}|a\rangle_{11}\left\langle k\left|+i \eta \omega_{2}(t) e^{-i \delta t} b\right| a\right\rangle_{11}\langle k| \\
& +i \eta \omega_{3}(t) e^{i \delta t} b|a\rangle_{22}\left\langle l\left|+i \eta \omega_{4}(t) e^{i \delta t} b^{\dagger}\right| a\right\rangle_{22}\langle k|+\text { H.c. }
\end{aligned}
$$

where $b$ and $b^{\dagger}$ are the annihilation and creation operators of the vibrational mode and $\eta$ is the Lamb-Dicke parameter that satisfies $\eta^{2}\left(n_{v}+1\right) \ll 1$ with $n_{v}$ being the quantum number of the vibrational mode. If the large detuning condition $|\delta| \gg\left|\eta \omega_{j}(t)\right|$ is satisfied, the single-ion transitions $|k\rangle \leftrightarrow|a\rangle$ and $|l\rangle \leftrightarrow|a\rangle$ are strongly suppressed, whereas only the double-ion transitions $|k l\rangle \leftrightarrow|a a\rangle$ and $|k k\rangle \leftrightarrow|a a\rangle$ are allowed due to vibrational energy exchanging between two ions. By using the approach in Ref. [62], one can further reduce the Hamiltonian in Eq. (A1). Specifically, it was proved in Ref. [62] that the effective Hamiltonian corresponding to the interaction one $H(t)=\Sigma_{n=1}^{N}\left(\hat{h}_{n} e^{-i \lambda_{n} t}+\hat{h}_{n}^{\dagger} e^{i \lambda_{n} t}\right)$ is $H_{\mathrm{eff}}(t)=\Sigma_{m, n=1}^{N} \frac{1}{2 \hbar}\left(\frac{1}{\lambda_{m}}+\frac{1}{\lambda_{n}}\right)\left[\hat{h}_{m}^{\dagger}, \hat{h}_{n}\right] e^{i\left(\lambda_{m}-\lambda_{n}\right) t}$. With $\hat{h}_{1}=$ $i \eta \omega_{1}(t) b^{\dagger}|a\rangle_{11}\left\langle k\left|, \quad \hat{h}_{2}=i \eta \omega_{2}(t) b\right| a\right\rangle_{11}\langle k|, \quad \hat{h}_{3}=-i \eta \omega_{3}^{*}(t) b^{\dagger}$ $|l\rangle_{22}\left\langle a\left|, \hat{h}_{4}=-i \eta \omega_{4}^{*}(t) b\right| k\right\rangle_{22}\langle a|$, and $\lambda_{1}=\lambda_{2}=\lambda_{3}=\lambda_{4}=$ $\delta$, one obtains the following effective Hamiltonian:

$$
H_{\text {eff }}(t)=\Omega_{k l}(t)|a a\rangle\left\langle k l\left|+\Omega_{k k}(t)\right| a a\right\rangle\langle k k|+\text { H.c. },
$$

where $\Omega_{k l}(t)=-\eta^{2} \omega_{1}(t) \omega_{3}(t) / \delta$ and $\Omega_{k k}(t)=\eta^{2} \omega_{2}(t) \omega_{4}$ $(t) / \delta$.

We also make use of a second form of the coupling mechanism with a similar procedure. Here, a pair of laser beams, one with detuning $-(v+\delta)$ and the other with detuning $(v-\delta)$, are applied to drive the transition $|k\rangle \leftrightarrow|a\rangle$ of ion 1; another pair of laser beams, one with detuning $-(v+\delta)$ and the other with detuning $(v-\delta)$, are applied to respectively drive the transitions $|l\rangle \leftrightarrow|a\rangle$ and $|k\rangle \leftrightarrow|a\rangle$. Under the large detuning condition and with the derivation similar to that in the previous paragraph, one obtains the effective Hamiltonian $H_{\text {eff }}^{\prime}(t)=\Omega_{a l}(t)|k a\rangle\left\langle a l\left|+\Omega_{a k}(t)\right| k a\right\rangle\langle a k|+$ H.c.

\section{APPENDIX B: RELEVANT HAMILTONIANS}

We now demonstrate the relevant Hamiltonians used in realizing holonomic multiqubit controlled phase flip gate. Taking these Hamiltonians as a reference, one obtains the relevant Hamiltonians used in other realization procedures, including the realization of a holonomic multiqudit gate. We consider the case where there are two blocks $k=1,2$, each having six ions with each ion encoding a qubit. We label the ions as $1, \ldots, 6$ in each block. For block $k=1$, to implement the transitions $|11\rangle_{1,2} \rightarrow\left|a_{0} a_{0}\right\rangle_{1,2}$ and $|01\rangle_{3,4} \rightarrow\left|a_{1} a_{1}\right\rangle_{3,4}$, we use the Hamiltonian $H(t)=\Omega_{1,2}(t)\left|a_{0} a_{0}\right\rangle_{1,2}\langle 11|+$ $\Omega_{3,4}(t)\left|a_{1} a_{1}\right\rangle_{3,4}\langle 01|+$ H.c. and the evolution time $T_{1}$ satisfies $\quad \int_{0}^{T_{1}}\left|\Omega_{1,2}(t)\right| d t=\int_{0}^{T_{1}}\left|\Omega_{3,4}(t)\right|=\pi / 2 . \quad$ The transitions $\quad\left|a_{0} 1\right\rangle_{2,4} \rightarrow\left|1 a_{0}\right\rangle_{2,4}$ and $|01\rangle_{5,6} \rightarrow\left|a_{1} a_{1}\right\rangle_{5,6}$ are implemented by using the Hamiltonian $H(t)=$ $\Omega_{2,4}(t)\left|1 a_{0}\right\rangle_{2,4}\left\langle a_{0} 1\left|+\Omega_{5,6}(t)\right| a_{1} a_{1}\right\rangle_{5,6}\langle 01|+$ H.c. and evolution time $T_{2}$ satisfying $\int_{0}^{T_{2}}\left|\Omega_{2,4}(t)\right| d t=\int_{0}^{T_{2}}\left|\Omega_{5,6}(t)\right|=\pi / 2$. Finally, we implement the transition $\left|a_{0} 1\right\rangle_{4,6} \rightarrow\left|1 a_{0}\right\rangle_{4,6}$ by using the Hamiltonian $H(t)=\Omega_{4,6}(t)\left|1 a_{0}\right\rangle_{4,6}\left\langle a_{0} 1\right|+$ H.c. with evolution time $T_{3}$ satisfying $\int_{0}^{T_{3}}\left|\Omega_{4,6}(t)\right|=\pi / 2$. For block $k=2$, to implement the transitions $|11\rangle_{1,2} \rightarrow$ $\left|a_{0} a_{0}\right\rangle_{1,2}, \quad|01\rangle_{3,4} \rightarrow\left|a_{1} a_{1}\right\rangle_{3,4}, \quad$ and $\quad|0\rangle_{6} \rightarrow\left|a_{1}\right\rangle_{6}$, we can use the Hamiltonian $H(t)=\Omega_{1,2}^{\prime}(t)\left|a_{0} a_{0}\right\rangle_{1,2}\langle 11|+$ $\Omega_{3,4}^{\prime}(t)\left|a_{1} a_{1}\right\rangle_{3,4}\left\langle 01\left|+\Omega_{6}^{\prime}(t)\right| a_{1}\right\rangle_{6}\langle 0|+$ H.c. with the evolution time $T_{1}^{\prime}$ satisfying $\int_{0}^{T_{1}^{\prime}}\left|\Omega_{1,2}^{\prime}(t)\right| d t=\int_{0}^{T_{1}^{\prime}} \mid \Omega_{3,4}^{\prime}$ $(t)\left|d t=\int_{0}^{T_{1}}\right| \Omega_{6}^{\prime}(t) \mid d t=\pi / 2$. The Hamiltonians used in the second and third steps of block $k=2$ are respectively the same as those used in the second and third steps of block $k=1$. The above operations cost three steps. After the above operations, we couple the two blocks by implementing the transition $\left|a_{0} a_{0}\right\rangle_{6_{1}, 6_{2}} \rightarrow-\left|a_{0} a_{0}\right\rangle_{6_{1}, 6_{2}}$, where state $|00\rangle_{6_{1}, 6_{2}}$ is the intermediate state and we have used the notation $\mu_{k}$ to denote ion $\mu$ of block $k$. To implement this operation, we use the Hamiltonian $H(t)=\Omega_{6_{1}, 6_{2}}(t)|00\rangle_{6_{1}, 6_{2}}\left\langle a_{0} a_{0}\right|+$ H.c. 
with the evolution time satisfying $\int_{0}^{T_{4}}\left|\Omega_{6_{1}, 6_{2}}(t)\right|=\pi$. We thereafter repeat the operations of the first three steps, but with both the implementation order and the implemented transitions reversed, which completes the realization. The used Hamiltonians are the same as those used in the first three steps. All the above-mentioned Hamiltonians can be realized by using the two coupling mechanisms introduced in the previous subsection. It is noteworthy that these Hamiltonians always couple states in the computational space with auxiliary states during the gate realization procedure. This fact guarantees that the realized gate is purely holonomic.
[1] M. A. Nielsen and I. L. Chuang, Quantum Computation and Quantum Information (Cambridge University Press, Cambridge, UK, 2001).

[2] P. W. Shor, Polynomial-time algorithms for prime factorization and discrete logarithms on a quantum computer, SIAM J. Comput. 26, 1484 (1997).

[3] L. K. Grover, Quantum Mechanics Helps in Searching for a Needle in a Haystack, Phys. Rev. Lett. 79, 325 (1997).

[4] E. Sjöqvist, D. M. Tong, L. M. Andersson, B. Hessmo, M. Johansson, and K. Singh, Non-adiabatic holonomic quantum computation, New J. Phys. 14, 103035 (2012).

[5] G. F. Xu, J. Zhang, D. M. Tong, E. Sjöqvist, and L. C. Kwek, Nonadiabatic Holonomic Quantum Computation in Decoherence-Free Subspaces, Phys. Rev. Lett. 109, 170501 (2012).

[6] J. Anandan, Non-adiabatic non-abelian geometric phase, Phys. Lett. A 133, 171 (1988).

[7] J. Zhang, L. C. Kwek, E. Sjöqvist, D. M. Tong, and P. Zanardi, Quantum computation in noiseless subsystems with fast nonAbelian holonomies, Phys. Rev. A 89, 042302 (2014).

[8] Z. T. Liang, Y. X. Du, W. Huang, Z. Y. Xue, and H. Yan, Nonadiabatic holonomic quantum computation in decoherence-free subspaces with trapped ions, Phys. Rev. A 89, 062312 (2014).

[9] Z. Y. Xue, J. Zhou, and Z. D. Wang, Universal holonomic quantum gates in decoherence-free subspace on superconducting circuits, Phys. Rev. A 92, 022320 (2015).

[10] C. F. Sun, G. C. Wang, C. F. Wu, H. D. Liu, X. L. Feng, J. L. Chen, and K. Xue, Non-adiabatic holonomic quantum computation in linear system-bath coupling, Sci. Rep. 6, 20292 (2016).

[11] Z. N. Zhu, T. Chen, X. D. Yang, J. Bian, Z. Y. Xue, and X. H. Peng, Single-Loop and Composite-Loop Realization of Nonadiabatic Holonomic Quantum Gates in a DecoherenceFree Subspace, Phys. Rev. Appl 12, 024024 (2019).

[12] B. J. Liu, X. K. Song, Z. Y. Xue, X. Wang, and M. H. Yung, Plug-and-Play Approach to Nonadiabatic Geometric Quantum Gates, Phys. Rev. Lett. 123, 100501 (2019).

[13] A. A. Abdumalikov, J. M. Fink, K. Juliusson, M. Pechal, S. Berger, A. Wallraff, and S. Filipp, Experimental realization of non-Abelian non-adiabatic geometric gates, Nature (London) 496, 482 (2013).

[14] G. R. Feng, G. F. Xu, and G. L. Long, Experimental Realization of Nonadiabatic Holonomic Quantum Computation, Phys. Rev. Lett. 110, 190501 (2013).

[15] S. Arroyo-Camejo, A. Lazariev, S. W. Hell, and G. Balasubramanian, Room temperature high-fidelity holonomic single-qubit gate on a solid-state spin, Nat. Commun. 5, 4870 (2014).

[16] C. Zu, W. B. Wang, L. He, W. G. Zhang, C. Y. Dai, F. Wang, and L. M. Duan, Experimental realization of universal geometric quantum gates with solid-state spins, Nature (London) 514, 72 (2014).

[17] J. Zhang, T. H. Kyaw, D. M. Tong, E. Sjöqvist, and L. C. Kwek, Fast non-Abelian geometric gates via transitionless quantum driving, Sci. Rep. 5, 18414 (2015).

[18] G. F. Xu, C. L. Liu, P. Z. Zhao, and D. M. Tong, Nonadiabatic holonomic gates realized by a single-shot implementation, Phys. Rev. A 92, 052302 (2015).

[19] E. Sjöqvist, Nonadiabatic holonomic single-qubit gates in offresonant $\Lambda$ systems, Phys. Lett. A 380, 65 (2016).

[20] E. Herterich and E. Sjöqvist, Single-loop multiple-pulse nonadiabatic holonomic quantum gates, Phys. Rev. A 94, 052310 (2016).

[21] Y. Wang, J. Zhang, C. Wu, J. Q. You, and G. Romero, Holonomic quantum computation in the ultrastrong-coupling regime of circuit QED, Phys. Rev. A 94, 012328 (2016).

[22] Z. Y. Xue, F. L. Gu, Z. P. Hong, Z. H. Yang, D. W. Zhang, Y. Hu, and J. Q. You, Nonadiabatic Holonomic Quantum Computation with Dressed-State Qubits, Phys. Rev. Appl 7, 054022 (2017).

[23] H. Li, Y. Liu, and G. L. Long, Experimental realization of single-shot nonadiabatic holonomic gates in nuclear spins, Sci. Chin.-Phys. Mech. Astron. 60, 080311 (2017).

[24] Y. Sekiguchi, N. Niikura, R. Kuroiwa, H. Kano, and H. Kosaka, Optical holonomic single quantum gates with a geometric spin under a zero field, Nat. Photon. 11, 309 (2017).

[25] B. B. Zhou, P. C. Jerger, V. O. Shkolnikov, F. J. Heremans, G. Burkard, and D. D. Awschalom, Holonomic Quantum Control by Coherent Optical Excitation in Diamond, Phys. Rev. Lett. 119, 140503 (2017).

[26] Z. P. Hong, B. J. Liu, J. Q. Cai, X. D. Zhang, Y. Hu, Z. D. Wang, and Z. Y. Xue, Implementing universal nonadiabatic holonomic quantum gates with transmons, Phys. Rev. A 97, 022332 (2018).

[27] S. Danilin, A. Vepsäläinen, and G. S. Paraoanu, Experimental state control by fast non-Abelian holonomic gates with a superconducting qutrit, Phys. Scr. 93, 055101 (2018).

[28] Y. Xu, W. Cai, Y. Ma, X. Mu, L. Hu, T. Chen, H. Wang, Y. P. Song, Z. Y. Xue, Z. Q. Yin, and L. Sun, Single-Loop Realization of Arbitrary Nonadiabatic Holonomic Single-Qubit Quantum Gates in a Superconducting Circuit, Phys. Rev. Lett. 121, 110501 (2018).

[29] P. Z. Zhao, G. F. Xu, and D. M. Tong, Nonadiabatic holonomic multiqubit controlled gates, Phys. Rev. A 99, 052309 (2019).

[30] T. X. Yan, B. J. Liu, K. Xu, C. Song, S. Liu, Z. S. Zhang, H. Deng, Z. G. Yan, H. Rong, K. Q. Huang, M. H. Yung, Y. Z. Chen, and D. P. Yu, Experimental Realization of Nonadiabatic Shortcut to Non-Abelian Geometric Gates, Phys. Rev. Lett. 122, 080501 (2019).

[31] Z. X. Zhang, P. Z. Zhao, T. H. Wang, L. Xiang, Z. L. Jia, P. Duan, D. M. Tong, Y. Yin, and G. P. Guo, Single-shot 
realization of nonadiabatic holonomic gates with a superconducting Xmon qutrit, New J. Phys. 21, 073024 (2019).

[32] N. Ramberg and E. Sjöqvist, Environment-Assisted Holonomic Quantum Maps, Phys. Rev. Lett. 122, 140501 (2019).

[33] M. Z. Ai, S. Li, Z. B. Hou, R. He, Z. H. Qian, Z. Y. Xue, J. M. Cui, Y. F. Huang, C. F. Li, and G. C. Guo, Experimental Realization of Nonadiabatic Holonomic Single-Qubit Quantum Gates with Optimal Control in a Trapped Ion, Phys. Rev. Appl 14, 054062 (2020).

[34] V. A. Mousolou, C. M. Canali, and E. Sjöqvist, Universal nonadiabatic holonomic gates in quantum dots and single-molecule magnets, New J. Phys. 16, 013029 (2014).

[35] B. P. Lanyon, M. Barbieri, M. P. Almeida, T. Jennewein, T. C. Ralph, K. J. Resch, G. J. Pryde, J. L. O'Brien, A. Gilchrist, and A. G. White, Simplifying quantum logic using higherdimensional Hilbert spaces, Nat. Phys. 5, 134 (2009).

[36] A. Fedorov, L. Steffen, M. Baur, M. P. da Silva, and A. Wallraff, Implementation of a Toffoli gate with superconducting circuits, Nature (London) 481, 170 (2012).

[37] M. Neeley, M. Ansmann, R. C. Bialczak, M. Hofheinz, E. Lucero, A. D. O'Connell, D. Sank, H. Wang, J. Wenner, A. N. Cleland, M. R. Geller, and J. M. Martinis, Emulation of a quantum spin with a superconducting phase qudit, Science $\mathbf{3 2 5}$, 722 (2009).

[38] M. J. Peterer, S. J. Bader, X. Jin, F. Yan, A. Kamal, T. J. Gudmundsen, P. J. Leek, T. P. Orlando, W. D. Oliver, and S. Gustavsson, Coherence and Decay of Higher Energy Levels of a Superconducting Transmon Qubit, Phys. Rev. Lett. 114, 010501 (2015).

[39] E. Svetitsky, H. Suchowski, R. Resh, Y. Shalibo, J. M. Martinis, and N. Katz, Hidden two-qubit dynamics of a four-level Josephson circuit, Nat. Commun. 5, 5617 (2014).

[40] M. Kues, C. Reimer, P. Roztocki, L. R. Cortés, S. Sciara, B. Wetzel, Y. Zhang, A. Cino, S. T. Chu, B. E. Little, D. J. Moss, L. Caspani, J. Azaña, and R. Morandotti, On-chip generation of high-dimensional entangled quantum states and their coherent control, Nature (London) 546, 622 (2017).

[41] C. Godfrin, A. Ferhat, R. Ballou, S. Klyatskaya, M. Ruben, W. Wernsdorfer, and F. Balestro, Operating Quantum States in Single Magnetic Molecules: Implementation of Grover's Quantum Algorithm, Phys. Rev. Lett. 119, 187702 (2017).

[42] E. O. Kiktenko, A. S. Nikolaeva, P. Xu, G. V. Shlyapnikov, and A. K. Fedorov, Scalable quantum computing with qudits on a graph, Phys. Rev. A 101, 022304 (2020).

[43] P. J. Low, B. M. White, A. A. Cox, M. L. Day, and C. Senko, Practical trapped-ion protocols for universal quditbased quantum computing, Phys. Rev. Research 2, 033128 (2020).

[44] R. Bianchetti, S. Filipp, M. Baur, J. M. Fink, C. Lang, L. Steffen, M. Boissonneault, A. Blais, and A. Wallraff, Control and Tomography of a Three Level Superconducting Artificial Atom, Phys. Rev. Lett. 105, 223601 (2010).

[45] F. Dolde, V. Bergholm, Y. Wang, I. Jakobi, B. Naydenov, S. Pezzagna, J. Meijer, F. Jelezko, P. Neumann, T. S. Herbrüggen, J. Biamonte, and J. Wrachtrup, High-fidelity spin entanglement using optimal control, Nat. Commun. 5, 3371 (2014).
[46] C. Senko, P. Richerme, J. Smith, A. Lee, I. Cohen, A. Retzker, and C. Monroe, Realization of a Quantum Integer-Spin Chain with Controllable Interactions, Phys. Rev. X 5, 021026 (2015).

[47] R. K. Naik, N. Leung, S. Chakram, P. Groszkowski, Y. Lu, N. Earnest, D. C. McKay, J. Koch, and D. I. Schuster, Random access quantum information processors using multimode circuit quantum electrodynamics, Nat. Commun. 8, 1904 (2017).

[48] P. Imany, J. A. Jaramillo-Villegas, M. S. Alshaykh, J. M. Lukens, O. D. Odele, A. J. Moore, D. E. Leaird, M. Qi, and A. M. Weiner, High-dimensional optical quantum logic in large operational spaces, npj Quantum Inf. 5, 59 (2019).

[49] Y. H. Luo, H. S. Zhong, M. Erhard, X. L. Wang, L. C. Peng, M. Krenn, X. Jiang, L. Li, N. L. Liu, C. Y. Lu, A. Zeilinger, and J. W. Pan, Quantum Teleportation in High Dimensions, Phys. Rev. Lett. 123, 070505 (2019).

[50] C. S. Wang, J. C. Curtis, B. J. Lester, Y. Zhang, Y. Y. Gao, J. Freeze, V. S. Batista, P. H. Vaccaro, I. L. Chuang, L. Frunzio, L. Jiang, S. M. Girvin, and R. J. Schoelkopf, Efficient Multiphoton Sampling of Molecular Vibronic Spectra on a Superconducting Bosonic Processor, Phys. Rev. X 10, 021060 (2020).

[51] K. Mølmer and A. Sørensen, Multiparticle Entanglement of Hot Trapped Ions, Phys. Rev. Lett. 82, 1835 (1999).

[52] A. Sørensen and K. Mølmer, Quantum Computation with Ions in Thermal Motion, Phys. Rev. Lett. 82, 1971 (1999).

[53] E. Moreno-Pineda, C. Godfrin, F. Balestro, W. Wernsdorfer, and M. Ruben, Molecular spin qudits for quantum algorithms, Chem. Soc. Rev. 47, 501 (2018).

[54] M. Erhard, R. Fickler, M. Krenn, and A. Zeilinger, Twisted photons: New quantum perspectives in high dimensions, Light: Sci. Appl. 7, 17146 (2018).

[55] Y. C. Wang, Z. X. Hu, B. C. Sanders, and S. Kais, Qudits and high-dimensional quantum computing, Front. Phys. 8, 589504 (2020).

[56] P. Gokhale, J. M. Baker, C. Duckering, N. C. Brown, K. R. Brown, and F. T. Chong, Asymptotic improvements to quantum circuits via qutrits, in ISCA '19: Proceedings of the 46th International Symposium on Computer Architecture (ACM, New York, 2019), pp. 554-566.

[57] E. T. Campbell, H. Anwar, and D. E. Browne, Magic-State Distillation in all Prime Dimensions Using Quantum Reed-Muller Codes, Phys. Rev. X 2, 041021 (2012).

[58] E. T. Campbell, Enhanced Fault-Tolerant Quantum Computing in $d$-Level Systems, Phys. Rev. Lett. 113, 230501 (2014).

[59] R. S. Andrist, J. R. Wootton, and H. G. Katzgraber, Error thresholds for Abelian quantum double models: Increasing the bit-flip stability of topological quantum memory, Phys. Rev. A 91, 042331 (2015).

[60] M. H. Michael, M. Silveri, R. T. Brierley, V. V. Albert, J. Salmilehto, L. Jiang, and S. M. Girvin, New Class of Quantum Error-Correcting Codes for a Bosonic Mode, Phys. Rev. X 6, 031006 (2016).

[61] S. Muralidharan, C. L. Zou, L. Li, J. Wen, and L. Jiang, Overcoming erasure errors with multilevel systems, New J. Phys. 19, 013026 (2017).

[62] D. F. V. James and J. Jerke, Effective Hamiltonian theory and its applications in quantum information, Can. J. Phys. 85, 625 (2007). 